\title{
The validity and reliability of computer-aided semen analyzers in performing semen analysis: a systematic review
}

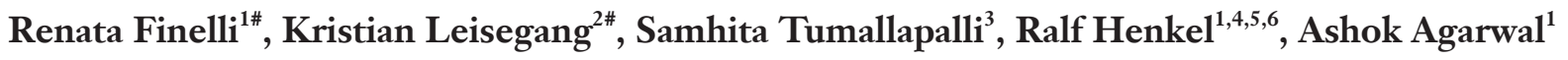 \\ ${ }^{1}$ American Center for Reproductive Medicine, Cleveland Clinic, Cleveland, OH, USA; ${ }^{2}$ School of Natural Medicine, Faculty of Community \\ and Health Sciences, University of the Western Cape, Bellville, South Africa; ${ }^{3}$ Case Western University, Cleveland, Ohio, USA; ${ }^{4}$ Department of \\ Metabolism, Digestion and Reproduction, Imperial College London, London, UK; ${ }^{5}$ Department of Medical Bioscience, University of the Western \\ Cape, Bellville, South Africa; ${ }^{6}$ LogixX Pharma, Theale, Berkshire, UK \\ Contributions: (I) Conception and design: R Finelli, K Leisegang, R Henkel, A Agarwal; (II) Administrative support: R Henkel, A Agarwal; (III) \\ Provision of study materials or patients: not applicable; (IV) Collection and assembly of data: R Finelli, K Leisegang, S Tumallapalli; (V) Data analysis \\ and interpretation: R Finelli. K Leisegang, S Tumallapalli; (VI) Manuscript writing: All authors; (VII) Final approval of manuscript: All authors. \\ "These authors contributed equally to this work. \\ Correspondence to: Ashok Agarwal, PhD, HCLD. Professor, Lerner College of Medicine, Director, Andrology Center, and American Center for \\ Reproductive Medicine, Cleveland Clinic, Mail Code X-11, 10681 Carnegie Avenue, Cleveland, OH 44195, USA. Email: agarwaa@ccf.org.
}

Background: Computer-aided sperm analyzers (CASA) are currently used worldwide for semen analysis. However, there are doubts about their reliability to fully substitute the human operator. Therefore, this study aimed to systematically review the current literature comparing results from semen evaluation by both CASA-based and manual approaches.

Methods: A systematic screening of the literature was performed based on the PRISMA guidelines and by searching on PubMed, Scopus, and Embase databases.

Results: A total of 14 studies were included. Our results showed a high degree of correlation for sperm concentration and motility when analysis was performed either manually or by using a CASA system. However, CASA results showed increased variability in low $(<15 \mathrm{million} / \mathrm{mL})$ and high $(>60 \mathrm{million} /$ $\mathrm{mL}$ ) concentration specimens, while sperm motility assessment was inaccurate in samples with higher concentration or in the presence of non-sperm cells and debris. Morphology results showed the highest level of difference, due to the high amount of heterogeneity seen between the shapes of the spermatozoa either in one sample or across multiple samples from the same subject.

Conclusions: Overall, our study suggests CASA systems as a valid alternative for the evaluation of semen parameters in clinical practice, especially for sperm concentration and motility. However, further technological improvements are required before these devices can one day completely replace the human operator. Artificial intelligence-based CASA devices promise to offer higher efficiency of the analysis and improve the reliability of results.

Keywords: Computer-aided sperm analyzers (CASA); computer-assisted sperm analysis; semen analysis; sperm concentration

Submitted Apr 02, 2021. Accepted for publication May 27, 2021.

doi: $10.21037 /$ tau-21-276

View this article at: http://dx.doi.org/10.21037/tau-21-276

\section{Introduction}

Infertility is defined as the inability to achieve a clinical pregnancy following one year of regular and timed intercourse without contraception (1). It is estimated that infertility affects approximately $15 \%$ of couples globally, where $50 \%$ of these cases are due to male infertility factors (2). For the clinical investigation of the male partner fertility potential, the standard semen analysis based on 




Microptic SCA



Microptic SCA SCOPE



Hamilton Thorne IVOS II



Hamilton Thorne CEROS ॥



MES SQA-Vision

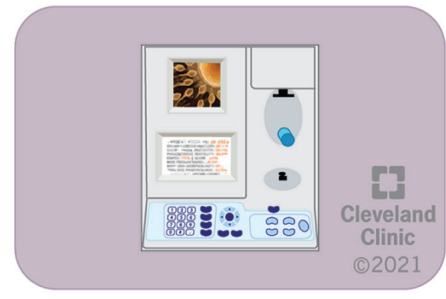

MES SQA-V Gold

Figure 1 Graphic representation of the most commonly CASA systems used in Andrology laboratories. MES, medical Electronic Systems; SCA, Sperm Class Analyzer; SQA, Sperm Quality Analyzer.

the WHO (2010) guidelines is considered the first line and cornerstone of investigation $(3,4)$. These guidelines provide laboratory procedures and reference thresholds for each semen parameter, including sperm concentration, motility, and morphology (4). As the evaluation is typically performed manually by a human operator, extensive training is required. However, the evaluation of microscopic parameters, including sperm concentration, motility, and morphology, is particularly prone to operator subjectivity, human error and intra-operator variability in results (5). These chances of error may affect the accuracy of results and therefore impact clinical decision making (5).

In the attempt to reduce variability and standardize the semen analysis, computer-aided sperm analyzers (CASA) have been used since the 1980s (6). CASA systems are automated instruments that use cameras and software to analyze data obtained by microscopic evaluation in order to provide semen parameter results (5). Initially, CASA systems were used mainly in the laboratory for research purposes. However, their use has spread into clinical practice as they reportedly reduce subjectivity and human error, and standardize the semen analysis process $(7,8)$. Moreover, they allow the analysis of a higher number of samples in less time, reducing the overall time needed, and increasing productivity (7). Hence, CASA systems have the potential to be used in laboratories to replace manual semen analysis (6). Over the last 40 years, the technological advancements have made these machines faster, smaller, and their results more accurate. Like any other "tech", these machines are constantly being updated with better software and hardware extensions (9).

There are several CASA systems that are currently available on the market (Figure 1). The Sperm Class Analyzer (SCA) produced by Microptics SL (Barcelona, Spain) measures semen concentration and motility by processing images obtained by phase-contrast microscopy. The SQA-V GOLD, produced by Medical Electronic Systems (Los Angeles, California, USA), uses electrooptics to track sperm concentration and motility. The IVOS and CEROS, produced by Hamilton-Thorne (Beverly, Massachusetts, USA), are image processing systems equipped with a microscope and camera $(6,10,11)$.

Although these and many other systems are increasingly being used globally, there remain concerns on their validity and reliability compared to the recommended manual semen analysis (5). The analysis of sperm morphology, for instance, is particularly challenging, as the shape of sperm may appear different at a microscopic examination depending on the plane where sperm are observed (12). Despite these limitations, a systematic analysis of the available evidence comparing semen results from both manual and CASA systems approaches is not available in the literature. Therefore, the aim of this study was to conduct a systematic review of recently published studies comparing 
or correlating results from CASA and manual semen analyses to determine the reliability of CASA use in clinical practice. We present the following article in accordance with the Preferred Reporting Items for Systematic Reviews and Meta-Analyses (PRISMA) guidelines (available at http://dx.doi.org/10.21037/tau-21-276).

\section{Methods}

\section{Ethical statement}

This study was performed by reviewing original studies published and retrieved by using different databases. An approval by the Institutional Review Board was, therefore, not required.

\section{Search strategy}

To identify eligible papers, a literature search was conducted on November 26, 2020, using the following keywords in PubMed, Scopus, and Embase databases: ("human" OR "men" OR "man" OR "patient*") AND ("CASA" OR "SQA" OR "SQA-V" OR "CellSoft" OR "HamiltonThorne" OR "Hamilton Thorne" OR "automated" OR "sperm class analy"” OR "computer-aided" OR "computerassisted" OR "computer assisted" OR "semi automated" OR "semi-automated" OR "SCA" OR "computer based" OR "computer-based") AND ("standard" OR "conventional" OR "routine" OR "manual") AND ("semen" OR "seminal" OR "sperm*"). Titles and/or abstracts of studies retrieved using this search strategy were screened independently by two review authors (S.T. and R.F.). The full text of these potentially eligible studies was then evaluated. Any disagreement between the authors over the eligibility of particular studies was resolved through discussion with a third author (KL). A pre-piloted form was used to extract data from the included studies for evidence synthesis.

\section{Study selection criteria}

Original English studies statistically comparing results from CASA- and manual-based semen analysis were included. Studies using latex Accu-Beads, validated quality control beads for personnel training, were also included. Only articles with results of manual semen analysis used as control for validation and studies that reported at least ONE of the following semen parameters were included: sperm concentration, total sperm count, total motility, progressive motility and/or sperm morphology. In order to include publications about the most updated CASA systems in the market, only articles published in the last 10 years (January 2010 to November 2020) were included. Reviews, meta-analysis, conference abstracts, posters, animal studies, and studies written in non-English languages were excluded.

\section{Results}

Through the application of the keyword search strategy, a total of 2,046 articles were retrieved. Duplicates $(n=447)$ and studies published before January $2010(\mathrm{n}=252)$ were removed, and 1,347 articles were screened based on title and abstract, with a further 1,175 non-relevant articles removed. Subsequently, the full-texts of the remaining 172 articles were screened for eligibility based on the inclusion and exclusion criteria, with a further 158 articles being removed. The inclusion process and reasons for exclusions are detailed in Figure 2. Finally, 14 articles were identified based on the inclusion criteria. The characteristics of each study are summarized in Table 1.

\section{Sperm concentration and total sperm count}

Ten articles compared sperm concentration results analyzed by both manual and CASA approaches. Vested $e t$ al. used a CRISMAS system to analyze semen samples from 166 young Danish men (mean age $=20$ years old). A significant difference in results was observed between CRISMAS and manual approach $(\mathrm{P}<0.001)$. According to the Bland-Altman plots, the differences between the two methods increased with increasing sperm concentration, with CRISMAS system overestimating sperm concentration by $7.7 \%$ (13). An overestimation of $14.7 \%$ was reported by TalarczykDesole et al., who analyzed 184 semen samples by using the SCA system (14).

On the contrary, Schubert et al. reported no significant difference in the results $(\mathrm{n}=150)$ when SCA system was used for analyzing either samples concentrated less than 1 million/mL or more than 80 million/mL (15). Also, results provided by SCA system showed higher repeatability in both oligo- and normozoospermic samples (15). Vernon et al. and Tomlinson et al. found no significant difference when using the SCA system and a novel CASA system using multi target-tracking algorithms to analyze the concentration of Accu-beads $(16,17)$. Similarly, comparable data was reported for both SQA V-GOLD and CEROS 


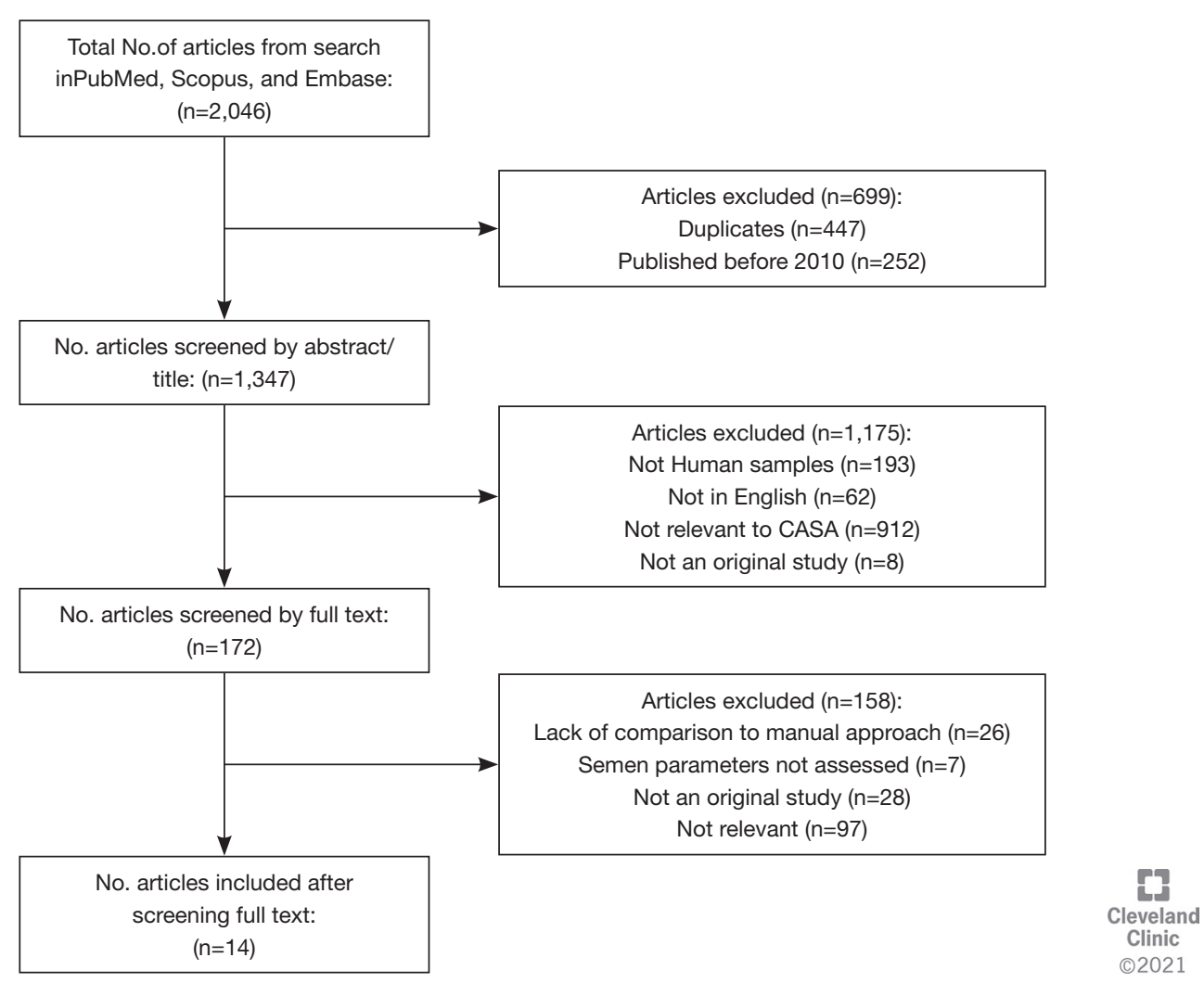

Figure 2 Workflow illustrating the screening process and reasons for exclusions.

Table 1 Characteristics of the articles included in this systematic review

\begin{tabular}{|c|c|c|c|}
\hline Reference & Sample characteristics and size & CASA system used & Main findings \\
\hline $\begin{array}{l}\text { Tomlinson } \\
\text { et al., } 2010\end{array}$ & $\begin{array}{l}\text { Semen samples }(n=287) \text { and } \\
\text { Accu-beads }\end{array}$ & $\begin{array}{l}\text { Novel CASA system using } \\
\text { multitarget-tracking algorithms }\end{array}$ & $\begin{array}{l}\text { High level of agreement and reproducibility for } \\
\text { measurement of sperm concentration and motility } \\
\text { between manual and CASA }(P<0.001)\end{array}$ \\
\hline $\begin{array}{l}\text { Vested et al., } \\
2011\end{array}$ & $\begin{array}{l}\text { Young Danish men } \\
(20.0 \pm 0.4 \text { years })(n=166)\end{array}$ & $\begin{array}{l}\text { CRISMAS clinical software version } \\
4.6 \text { (Image House Medical, } \\
\text { Copenhagen, Denmark) }\end{array}$ & $\begin{array}{l}\text { CRISMAS overestimated sperm concentration } \\
\text { and the percentage of rapidly progressive motility, } \\
\text { while underestimated the percentage of slowly } \\
\text { progressive and non-progressive motility }(P<0.001)\end{array}$ \\
\hline
\end{tabular}

Table 1 (continued) 
Table 1 (continued)

\begin{tabular}{|c|c|c|c|}
\hline Reference & Sample characteristics and size & CASA system used & Main findings \\
\hline $\begin{array}{l}\text { Dearing et al., } \\
2014\end{array}$ & Semen samples $(n=352)$ & SCA V 4.0 & $\begin{array}{l}\text { High correlation for the evaluation of sperm count } \\
(r=0.95) . \text { SCA overestimation in case of low sperm } \\
\text { count }\end{array}$ \\
\hline $\begin{array}{l}\text { Talarczyk- } \\
\text { Desole et al., } \\
2017\end{array}$ & Semen samples $(n=230)$ & SCA 5.4 & $\begin{array}{l}\text { Manual and SCA results differed }(\mathrm{P}<0.0001) \text { for } \\
\text { sperm concentration, progressive motility, and } \\
\text { morphology }\end{array}$ \\
\hline $\begin{array}{l}\text { Engel et al., } \\
2019\end{array}$ & Semen samples $(n=100)$ & SQA Vision & $\begin{array}{l}\text { Correlation between manual and SQA Vision } \\
\text { is higher for sperm concentration }(r=0.98) \text { than } \\
\text { progressive }(r=0.86) \text { and total motility }(r=0.74) \text {, and } \\
\text { morphology }(r=0.36)\end{array}$ \\
\hline $\begin{array}{l}\text { Dearing et al., } \\
2019\end{array}$ & Semen samples $(n=225)$ & SCA 4.1 & $\begin{array}{l}\text { Difference in evaluation of sperm motility between } \\
\text { manual and SCA analysis }(P<0.001) \text {. Correlation } \\
\text { reported only for "a" and "d" grade sperm motility } \\
(r=0.58, r=0.63 \text {, respectively; } P<0.001)\end{array}$ \\
\hline $\begin{array}{l}\text { Baig et al., } \\
2019\end{array}$ & Infertile men $(n=60)$ & $\begin{array}{l}\text { BIOVIS } 2000 \text { (Expert Vision Labs } \\
\text { Pvt. Limited, India) }\end{array}$ & $\begin{array}{l}\text { Sperm concentration analyzed manually and by } \\
\text { BIOVIS } 2000 \text { highly correlated }(r=0.99 ; P<0.0001)\end{array}$ \\
\hline
\end{tabular}

CASA, computer-aided sperm analyzers; SAIS, Sperm Analysis Imaging System; SEEM, Sperm self check kit \& smartphone app; SCA, Sperm Class Analyzer; SMAS, Sperm Motility Analyzer System; SQA, Sperm Quality Analyzer.

when compared with manual analysis (11). However, both automated systems showed a significant overestimation of sperm concentration $(\mathrm{P}<0.05)$ in patients with moderate oligozoospermia (sperm concentration $=5-15 \mathrm{million} / \mathrm{mL}$ ). This overestimation was also reported in severe oligozoospermic (sperm concentration $<5$ million/mL) patients by the CEROS system. Furthermore, statistically significant positive correlations between manual and CASAbased approaches were highlighted in several studies using different types of CASA systems, as reported in Table 2 (17-22).
Unlike other studies, Dearing et al. reported and compared total sperm count results obtained manually and by using a SCA system on a larger sample size of 352 patients. Authors observed a high correlation between CASA and manual results, regardless the type of counting chamber used (Neubauer: $r=0.94$; Leja: $r=0.92$; $\mathrm{P}<0.0001)$ (23). However, the correlation decreased in the groups of moderate and severe oligozoospermia, depending on the type of counting chamber (Neubauer: $r=0.64$ and 0.66 , respectively; Leja: $r=0.74$ and 0.56 , respectively) used. 
Table 2 Correlation coefficients and $\mathrm{P}$ values comparing sperm concentration assessment with CASA systems and manual method

\begin{tabular}{|c|c|c|c|}
\hline Reference & CASA System & Correlation Coefficient & $P$ value \\
\hline \multirow[t]{2}{*}{ Akashi et al., 2010} & CellSoft & 0.80 & $<0.0001$ \\
\hline & SMAS & 0.87 & \\
\hline Tomlinson et al., 2010 & Novel CASA system & 0.94 & $<0.001$ \\
\hline Baig et al., 2019 & BIOVIS 2000 & 0.99 & $<0.0001$ \\
\hline \multirow[t]{2}{*}{ Cheon et al., 2019} & $\begin{array}{l}\text { Smartphone-based CASA System (SEEM) (Recruit Lifestyle Co., Ltd., } \\
\text { Toyo, Japan) }\end{array}$ & 0.38 & 0.04 \\
\hline & Laboratory based CASA (SAIS plus) (Medical Supply, Seoul, Korea) & 0.99 & $<0.0001$ \\
\hline
\end{tabular}

CASA, computer-aided sperm analyzers; SAIS, Sperm Analysis Imaging System; SEEM, Sperm self check kit \& smartphone app; SCA, Sperm Class Analyzer; SMAS, Sperm Motility Analyzer System; SQA, Sperm Quality Analyzer.

\section{Total motility}

Five articles were included comparing results for total motility by manual and CASA analyses. Cheon et al., and Lammers $e t$ al. reported no significant difference between both approaches when results were globally analyzed $(11,21)$. However, in the latter study, the median for total motility with both SQA-V Gold and CEROS was significantly lower than the manual measurements in case of severe oligozoospermia ( $<5$ million $/ \mathrm{mL}$ ). Moderate oligozoospermia samples showed significantly lower measurements with only the CEROS system. Conversely, the median reported for normozoospermic samples was significantly higher than manual evaluation $(74.3 \%$ vs $62 \%$, $\mathrm{P}<0.05)$ when CEROS was used (11). Akashi et al. tested 81 infertile men samples using the CellSoft and SMAS systems and reported significant $(\mathrm{P}<0.0001)$ correlations for both systems with the manual analysis $(r=0.83$ and $r=0.69$, respectively) (19). Agarwal et al. found statistically significant differences between results when using the LensHooke X1PRO device $(\mathrm{P}<0.001)$, although they reported a high positive correlation coefficient $(r=0.93, \mathrm{P}<0.01)(22)$. High correlation coefficients were also reported when the manual method was compared to a laboratory-based CASA system $(\mathrm{r}=0.97 ; \mathrm{P}<0.0001)$ or a smartphone-based CASA analysis $(\mathrm{r}=0.59 ; \mathrm{P}<0.001)(21)$ as well as SQA Vision $(\mathrm{r}=0.74$; $\mathrm{P}<0.0001)(18)$.

\section{Progressive motility}

Seven articles compared results for progressive motility by analyzing samples manually or using $\operatorname{SCA}(15,18,24)$, LensHooke X1 PRO (22), SQA-Vision (16), SQA-V GOLD and CEROS (11), and an unnamed system (17). An agreement between both manual and SCA analysis was reported analyzing 30 semen samples (15). Particularly, authors reported a better repeatability when SCA was used for analyzing either astheno- or normozoospermic samples (15). No significant difference was reported also by Lammers et al. (2014) when using both the SQA V-GOLD and CEROS systems and similarly by Agarwal et al. when using the LensHooke X1 PRO (22). Conversely, the studies conducted by Dearing et al. and Talarczyk et al. reported a significant difference between the results $(\mathrm{P}<0.001$ and $\mathrm{P}<0.0001$, respectively) (14,24). A high correlation coefficient between manual and CASA-based analyses were reported when SQA Vision ( $\mathrm{r}=0.86$; $\mathrm{P}$ value not reported) (18), and a novel CASA system using a multitarget tracking algorithms $(\mathrm{r}=0.85 ; \mathrm{P}<0.0001)(17)$ were used.

\section{Morphology}

Of the five articles comparing results for sperm morphology by manual and CASA approaches, the CEROS and SQA V-GOLD $(\mathrm{P}<0.05)(11)$, and SCA $(\mathrm{P}<0.0001)(14)$, reported a significant difference. A low correlation between both methods was observed when using SQA vision $(r=0.36$; $\mathrm{P}$ value not reported) (18). Contrary to the abovementioned results, Singh et al. (25) reported a significant high correlation $(\mathrm{r}=0.77 ; \mathrm{P} \leq 0.001)$ by using the SQA 
Table 3 Pros and cons of using CASA systems for semen analysis in comparison with the manual method

\begin{tabular}{ll}
\hline Pros & Cons \\
\hline $\begin{array}{l}\text { Faster analysis of a large number of semen } \\
\text { samples }\end{array}$ & Trained personnel required \\
Reduced subjectivity & Analysis is challenging for viscous samples, presence of round cells, debris and agglutination \\
Higher reproducibility & Low accuracy in the analysis of sperm morphology \\
Evaluation of sperm motion characteristics & Challenging to compare results between different instruments \\
Possibility to record videos and pictures & Variability in sperm concentration analysis for samples which are very low or high concentrated
\end{tabular}

IIC-P for the semen evaluation of 201 infertile men, as well as high sensitivity (85.5\%), specificity (87.3\%) and positive predictive value (93.7\%). Moreover, results for sperm morphology analyzed by SCA were reported by Schubert et al. to be in agreement with those obtained by manual analysis (15). Finally, SCA analysis was associated with a lower variation in the results for either severely teratozoospermic or normozoospermic samples compared to manual semen analysis (15).

\section{Discussion}

Since the 1980s, CASA technology has been increasingly used in research and clinical practice, and in some cases, to substitute the manual semen analysis $(9,26)$. This review aimed to summarize the current evidence investigating the validity of commercial CASA systems as an alternative to the routine seminal analysis in the evaluation of semen quality. Through a systematic review of the relevant literature, these results suggest that parameters such as sperm concentration, total and progressive motility can be analyzed interchangeably and reliably by both CASA and manual approaches, while evaluation of sperm morphology by CASA systems correlates poorly with the manual morphology scores.

Sperm concentration tends to have a high correlation between both approaches for the various systems used. In 2 studies, authors reported a high correlation by using Accu-beads $(16,17)$. These are latex beads, commonly used for laboratory quality control or for training of junior technicians. As they are immotile, suspended in aqueous solution and well-characterized, it is not surprising that a high rate of correlation was observed between manual and CASA results. However, when semen samples with motile sperm were examined, the analyzers were not free of errors (Table 3). Variability in results have been highlighted when semen concentration was analyzed by computer-based analyzers in samples that were very low or high concentrated $(14,17)$. Generally, these analyzers slightly overestimate the sperm concentration, mainly due to the incorrect identification of other cellular types and debris as sperm cells $(11,13,14,23)$ (Figure 3). However, an underestimation due to the subjectivity of the manual approach and the evaluation of a limited number of spermatozoa in the case of oligozoospermia cannot be excluded. To compensate for this, some analyzers have been upgraded with specific filters for the identification of such debris, which are removed from the analysis (15). Also, samples with concentrations greater than 60 million/ mL must be diluted for accuracy. Once the sample is diluted, the same aliquot is loaded on the counting chamber and analyzed manually as well as by CASA system. The dilution factor is included in the final calculations for both techniques. There is no bias as the same sample that is diluted is counted both manually and by CASA device. The incorrect dilution of the sample may introduce a technical bias due to the use of uncalibrated pipettes, along with incorrect pipetting performed by the technician, or the viscous nature of some samples, significantly affecting the results. However this bias is identical for both manual and CASA device as it is the same sample that is analyzed (27).

An unreliable report of sperm concentration may lead to an incorrect evaluation of semen quality, with oligozoospermic patients being identified as normozoospermic, or oligozoospermia being defined as "moderate" instead of "severe". This may influence the clinical management of these patients, as the evaluation of semen quality is important in clinical diagnostics and management, including the type of ART technique to be used (IVF/ICSI or IUI). Hence, it would be of utmost clinical interest to identify the range of sperm concentration values which can be accurately detected by using a CASA 




Figure 3 Causes for error in semen analysis when a CASA system is used are summarized.

system. Lammers et al. reported both SQA-V Gold and CEROS system to significantly overestimate the sperm concentration when lower than $15 \mathrm{million} / \mathrm{mL}$ (11). This is in agreement with the study published by Dearing et al., which reported a similar overestimation at the same value of sperm concentration $(<15$ million/mL) (23). Although Tomlinson et al. reported a general agreement between results from manual and CASA analyses, this was less pronounced when sperm concentration was higher than 60 million/mL (17). Whereas, Vested et al. and Schubert et al. reported increasing differences in the results when sperm concentration analyzed was higher than 100 million/mL $(13,15)$. Therefore, the use of a CASA system for routine semen analysis is not accurate in samples which show very low $(<15$ million $/ \mathrm{mL})$ or high $(>100$ million $/ \mathrm{mL})$ sperm concentration.

A high correlation between manual and CASA-based approach was reported for sperm motility (15). This can be credited to the ease of identification and tracking of sperm movement. However, the difficulties of CASA systems in distinguishing between immotile sperm, non-sperm cells (NSCs), and debris can cause inaccurate evaluation of sperm motility and sperm count as well. To solve this issue, systems have been updated with better software recognition that can identify a 'blob' as either sperm or an NSC, leading to better results (9). Analysis of sperm motility may also be inaccurate when immotile cells are pushed on a path by a motile sperm cell. Here, immotile cells can be incorrectly read as motile cells, leading to an overestimation of sperm motility percentage (14). However, some studies reported 
an under-estimation of motility when an analyzer is used $(11,22)$. This may be explained by an overestimation of motile sperm when counted manually, as human eyes are attracted by the movement (28). Human eyes are also incapable of distinguishing between different types of sperm motility, whereas a camera and tracking system can easily recognize and classify the type of movement clearer and faster (17). This is of great clinical interest, as few studies reported a statistical correlation between sperm kinematics and reproductive outcomes, even though this remains controversial $(9,29,30)$.

In contrast to sperm concentration and motility, morphology is the most difficult parameter to analyze and the least reliable one to assess. This is due to heterogeneity between the shapes of the spermatozoa either in one sample or across multiple samples from the same subject (3). Abnormal spermatozoa are defined as sperm with deformities or defects at any part of its body. Therefore, the identification of all types of abnormality by a CASA machine is particularly challenging which explains the low correlation coefficients observed when comparing the two approaches. Besides the paucity of studies analyzing the performance of CASA systems for sperm morphology evaluation $(\mathrm{n}=5)$, a different classification system was used by Schubert et al. (15), who analyzed sperm morphology based on David's modified criteria (31), and not the strict criteria recommended by the WHO and used in the other studies (4). A poor correlation was reported between these two classification systems (32). We can assume that any device can "learn" how to classify sperm morphology based on different criteria. However, this represents an additional challenge to compare studies using different classification systems, and increases the uncertainty about the real capacity of a CASA system to classify sperm morphology correctly.

There are further concerns when considering use of automated systems in the semen analysis (Table 3). As with the manual semen analysis, laboratory errors in the CASA evaluation might still be introduced by the human operator in systems that require a sample to be manually mixed and loaded to fill the chamber. This may be due to improper mixing of the sample underlying pipetting errors or diluting errors, influencing the software analysis and leading to inaccurate results. Furthermore, the systems require routine cleaning and calibration. Hence, proficient, and well-trained personnel are still required when using CASA systems for analysis (9). The use of CASA systems was also seen to be limited by samples that have high sperm agglutination (17). On the other side, some CASA systems (i.e., IVOS II) are equipped with heated specimen stage, which allows precise control of temperature during the analysis, constant to within $0.5^{\circ} \mathrm{C}$, whereas manual semen analysis is usually conducted under a phase-contrast microscope at room temperature. This represents an unquestionable advantage, as changes in temperature may significantly affect the analysis of sperm parameters, particularly motility assessment. Finally, using a computer-based analyzer allows fast processing of a larger number of samples in less time. Therefore, the purchase of this instrument could be convenient for the laboratories that process large volume of samples.

Despite all the above-mentioned considerations, this systematic review of the literature assumes that the manual semen analysis is the gold standard. The manual semen analysis is considered the gold standard when performed by highly trained competent technologists working in accredited lab and participating in a proficiency testing program monitored by external agencies. However, large variability has been reported between different operators or laboratories analyzing the semen parameters due to the lack of an external quality control program, not following the standardized WHO procedures, or lack of appropriately trained technologies (33-35). Therefore, the control used to compare CASA systems performance may be itself inaccurate and show great deal of variability, and this represents a limitation for our analysis.

As the technology is evolving continuously, artificial intelligence (AI)-based devices promise to improve the efficiency of the analysis and the reliability of the results. In the Andrology laboratory, the LensHooke X1 PRO represents the most recently developed device which integrates AI algorithms and autofocus optical technology for the analysis of semen parameters. In comparison with the existing CASA systems, this device is compact, portable, easy-to-use, and results correlate highly with those obtained by the manual semen analysis. Although pilot study showed some differences in manual and CASA sperm motility (22), in a recent study the Lenshooke X1PRO has reportedly shown the ability to detect a very large range of sperm concentration (between 0.1-300 million/mL). Moreover, it demonstrated higher sensitivity and specificity $(>90 \%)$ in identifying oligo- and asthenozoospermic samples when compared to IVOS II system (36). Hence, further development of AI-based devices represents step in the right direction for the automation of semen analysis. 


\section{Conclusions}

This systematic analysis of the literature suggests CASA systems to be a valid alternative for the evaluation of semen parameters in clinical practice, especially when related to sperm concentration and motility measurements. However, its use is less reliable when sperm morphology is evaluated. Hence, further improvements in this technology are necessary and relevant validation studies should be conducted to establish the accuracy and precision of CASA test results before a human operator might be completely substituted by a CASA system for semen analysis.

\section{Acknowledgments}

Authors are thankful to the artists from the Cleveland Clinic's Center for Medical Art \& Photography for their help with the illustrations. Authors thank Dr. Rakesh Sharma and Dr. Sajal Gupta (Cleveland Clinic) for their critical feedback and support during the revision of this manuscript.

Funding: The study was supported by the American Center for Reproductive Medicine.

\section{Footnote}

Reporting Checklist: The authors have completed the PRISMA reporting checklist. Available at http://dx.doi. org/10.21037/tau-21-276

Peer Review File: Available at http://dx.doi.org/10.21037/ tau-21-276

Conflict of Interest: All authors have completed the ICMJE uniform disclosure form (available at http://dx.doi. org/10.21037/tau-21-276). The authors have no conflicts of interest to declare.

Ethical Statement: The authors are accountable for all aspects of the work in ensuring that questions related to the accuracy or integrity of any part of the work are appropriately investigated and resolved.

Open Access Statement: This is an Open Access article distributed in accordance with the Creative Commons Attribution-NonCommercial-NoDerivs 4.0 International License (CC BY-NC-ND 4.0), which permits the noncommercial replication and distribution of the article with the strict proviso that no changes or edits are made and the original work is properly cited (including links to both the formal publication through the relevant DOI and the license). See: https://creativecommons.org/licenses/by-nc-nd/4.0/.

\section{References}

1. Zegers-Hochschild F, Adamson GD, Dyer S, et al. The international glossary on infertility and fertility care, 2017. Hum Reprod 2017;32:1786-801.

2. Agarwal A, Mulgund A, Hamada A, et al. A unique view on male infertility around the globe. Reprod Biol Endocrinol 2015;13:37.

3. Baskaran S, Finelli R, Agarwal A, et al. Diagnostic value of routine semen analysis in clinical andrology. Andrologia 2021;53:e13614.

4. World Health Organization. Laboratory manual for the examination and processing of human semen. 5th Ed. Geneva, Switzerland; 2010.

5. Douglas C, Parekh N, Kahn LG, et al. A novel approach to improving the reliability of manual semen analysis: A paradigm shift in the workup of infertile men. World J Mens Health 2021;39:172-85.

6. Mortimer ST, Van Der Horst G, Mortimer D. The future of computer-aided sperm analysis. Asian J Androl 2015;17:545-53.

7. Amann RP, Waberski D. Computer-assisted sperm analysis (CASA): Capabilities and potential developments. Theriogenology 2014;81:5-17.e1.

8. Amann RP, Katz DF. Reflections on CASA after 25 years. J Androl 2004;25:317-25.

9. Tomlinson MJ, Naeem A. CASA in the medical laboratory: CASA in diagnostic andrology and assisted conception. Reprod Fertil Dev 2018;30:850-9.

10. Agarwal A, Sharma R. Automation is the key to standardized semen analysis using the automated SQA-V sperm quality analyzer. Fertil Steril 2007;87:156-62.

11. Lammers J, Splingart C, Barriere P, et al. Double-blind prospective study comparing two automated sperm analyzers versus manual semen assessment. J Assist Reprod Genet 2014;31:35-43.

12. Menkveld R, Holleboom C, Rhemrev J. Measurement and significance of sperm morphology. Asian J Androl 2011;13:59-68.

13. Vested A, Ramlau-Hansen CH, Bonde JP, et al. A comparison of conventional and computer-assisted semen analysis (CRISMAS software) using samples from 166 young Danish men. Asian J Androl 2011;13:453-8.

14. Talarczyk-Desole J, Berger A, Taszarek-Hauke G, et al. 
Manual vs. computer-assisted sperm analysis: Can CASA replace manual assessment of human semen in clinical practice? Ginekol Pol 2017;88:56-60.

15. Schubert B, Badiou M, Force A. Computer-aided sperm analysis, the new key player in routine sperm assessment. Andrologia 2019;51:e13417.

16. Vernon DD, Johnson JE, Houwing AM, et al. Accu-Beads as a quality control measure for manual and automated methods of measuring sperm concentration - An observational study. J Assist Reprod Genet 2014;31:25-33.

17. Tomlinson MJ, Pooley K, Simpson T, et al. Validation of a novel computer-assisted sperm analysis (CASA) system using multitarget-tracking algorithms. Fertil Steril 2010;93:1911-20.

18. Engel KM, Grunewald S, Schiller J, et al. Automated semen analysis by SQA Vision @ versus the manual approach-A prospective double-blind study. Andrologia 2019;51:e13149.

19. Akashi T, Watanabe A, Komiya A, et al. Evaluation of the Sperm Motility Analyzer System (SMAS) for the assessment of sperm quality in infertile men. Syst Biol Reprod Med 2010;56:473-7.

20. Baig A, Shoebuddin M, Ahmed M. Comparison of manual sperm analysis with computer-assisted sperm analysis: A comparative cross-sectional study. Natl J Physiol Pharm Pharmacol 2019;9:862-4.

21. Cheon WH, Park HJ, Park MJ, et al. Validation of a smartphone-based, computer-assisted sperm analysis system compared with laboratory-based manual microscopic semen analysis and computer-assisted semen analysis. Investig Clin Urol 2019;60:380-7.

22. Agarwal A, Henkel R, Huang CC, et al. Automation of human semen analysis using a novel artificial intelligence optical microscopic technology. Andrologia 2019;51:e13440.

23. Dearing CG, Kilburn S, Lindsay KS. Validation of the sperm class analyser CASA system for sperm counting in a busy diagnostic semen analysis laboratory. Hum Fertil (Camb) 2014;17:37-44.

24. Dearing C, Jayasena C, Lindsay K. Can the Sperm Class Analyser (SCA) CASA-Mot system for human sperm motility analysis reduce imprecision and operator subjectivity and improve semen analysis? Hum Fertil (Camb) 2021;24:208-18.

25. Singh S, Sharma S, Jain M, et al. Importance of papanicolaou staining for sperm morphologic analysis: comparison with an automated sperm quality analyzer. Am
J Clin Pathol 2011;136:247-51.

26. Knuth UA, Nieschlag E. Comparison of computerized semen analysis with the conventional procedure in 322 patients. Fertil Steril 1988;49:881-5.

27. Brito LFC, Althouse GC, Aurich C, et al. Andrology laboratory review: Evaluation of sperm concentration. Theriogenology 2016;85:1507-27.

28. Komori K, Tsujimura A, Ishijima S, et al. Comparative study of sperm motility analysis system and conventional microscopic semen analysis. Reprod Med Biol 2006;5:195-200.

29. Jędrzejczak P, Rzepczyńska I, Taszarek-Hauke G, et al. Effect of sperm subpopulation's kinetics on human fertilization in vitro. Arch Androl 2005;51:185-93.

30. Freour T, Jean M, Mirallie S, et al. Predictive value of CASA parameters in IUI with frozen donor sperm. Int J Androl 2009;32:498-504.

31. Auger J, Jouannet P, Eustache F. Another look at human sperm morphology. Hum Reprod 2016;31:10-23.

32. Blanchard M, Haguenoer K, Apert A, et al. Sperm morphology assessment using David's classification: Time to switch to strict criteria? Prospective comparative analysis in a selected IVF population. Int J Androl 2011;34:145-52.

33. Filimberti E, Degl'Innocenti S, Borsotti M, et al. High variability in results of semen analysis in andrology laboratories in Tuscany (Italy): the experience of an external quality control (EQC) programme. Andrology 2013;1:401-7.

34. Punjabi U, Wyns C, Mahmoud A, et al. Fifteen years of Belgian experience with external quality assessment of semen analysis. Andrology 2016;4:1084-93.

35. Brazil C, Swan SH, Tollner CR, et al. Quality control of laboratory methods for semen evaluation in a multicenter research study. J Androl 2004;25:645-56.

36. Agarwal A, Panner Selvam MK, Ambar RF. Validation of LensHooke® X1 PRO and computer-assisted semen analyzer compared with laboratory-based manual semen analysis. World J Mens Health. 2021;39. [Epub ahead of print]. doi: 10.5534/wjmh.200185.

Cite this article as: Finelli R, Leisegang K, Tumallapalli S, Henkel R, Agarwal A. The validity and reliability of computerassisted semen analyzers in performing semen analysis: a systematic review. Transl Androl Urol 2021;10(7):3069-3079. doi: 10.21037/tau-21-276 